\title{
Design Demonstration System of SIEMENS S7 Series PLC Network Based on PROFIBUS
}

\author{
Chang Ruokui \\ College of Engineering and Technology \\ Tianjin Agricultural University, \\ Jinjing Road 22, 300384, Tianjin, China \\ changrk@163.com
}

\author{
Zhang $\mathrm{Wu}$ \\ College of Engineering and Technology \\ Tianjin Agricultural University, \\ Jinjing Road 22, 300384, Tianjin, China \\ 1099884505@qq.com
}

\begin{abstract}
PLC principle and application is a kind of theory and practice, engineering practice of the professional course. Among them, PLC experimental teaching is an important part of the course. Many Chinese universities opened the PLC curriculum, teachers use experimental board to describe the whole process in PLC experiment teaching, the operation effect of the board is using light-emitting diode (LED) to simulate the. As a result, the running state of the experiment is very abstract, not intuitive, and it is difficult to feel the reality of the state, it is difficult to understand the students to absorb. The design was constructed to simulate real environment of the demo system, using the Siemens S7-400 PLC as the main station controller and PLC of Siemens S7-200/300 is from the station controller with EB8000 human-machine interface, PROFIBUS-DP communication, RFID technology and four degrees of freedom for the sorting machinery to achieve mail classification sorting monitoring. The system should be able to achieve different PLC hardware networking, communication and monitoring and other functions, so as to achieve the intuitive, flexible and participatory experimental teaching. The system solves the problem of the replacement of the controlled object, and the running process is vivid, and the reality is very strong. Therefore, students can be able to solve the PLC experiment to learn to be able to do the theory with practice.
\end{abstract}

Keywords—RFID technology; Programmable logic controller ; PROFIBUS-DP communication

\section{INTRODUCTION}

Programmable logic controller (PLC) is a new type of artificial intelligent control device combined with computer technology, remote communication and automatic control[1]. PLC technology is a very important technology in the field of automatic control in the current society. In today's society for the needs of this area, universities across the country with electronic, electrical and automation and other professional have PLC to join the university education and teaching as part of the required professional courses.

PLC control technology and application is a professional course, its theory and practice are equally important and engineering practice is strong. Promote in the university to students as the main participants, in an integrated design for the focus of practice teaching, to improve students' flexible using PLC theory application, to solve the problem and improve the design ability and creative ability training has an important significance.

At present, in our country, many universities open course of PLC, the teachers use control experiment board to tell the whole process control at the PLC experiment teaching explanation. The operation effect of the board is using lightemitting diode (LED) simulation. As a result, the running state of the experiment is very abstract, not intuitive, and it is difficult to feel and understand the reality of the state for the students. Eventually it led to the students can only learn knowledge and not to apply, just for it's very boring. Therefore, therefore, the project designs demonstration system to realize the simulation of the actual field control system. The system is using the Siemens S7-400 PLC as the main station controller and PLC of Siemens S7-200/300 is from the station controller with man-machine interface configuration software MCGS, PROFIBUS-DP communication, RFID technology and four degrees of freedom for the sorting machinery realize mail classification sorting monitoring demonstration[2]-[3]. The system solves the problem of the replacement of the controlled object, and the running process is vivid, and the reality is very strong. So it is able to improve students' interest in learning PLC experiments, so that the theory can be linked with practice.

\section{Design OF Simulation DEMONSTRATION SYSTEM}

\section{A. Hardware Design of Simulation Demonstration System}

The analog mail sorting system is mainly based on the man-machine interface technology, Internet international Internet technology and radio frequency identification technology to monitor the terminal and the station's mail sorting. The hardware is mainly composed of SIEMENS PLC, touch screen, 4 degrees of freedom sorting manipulator, sweep code instrument, PROFIBUS communication cable and stepping motor. Its system structure diagram is shown in Fig. 1. 


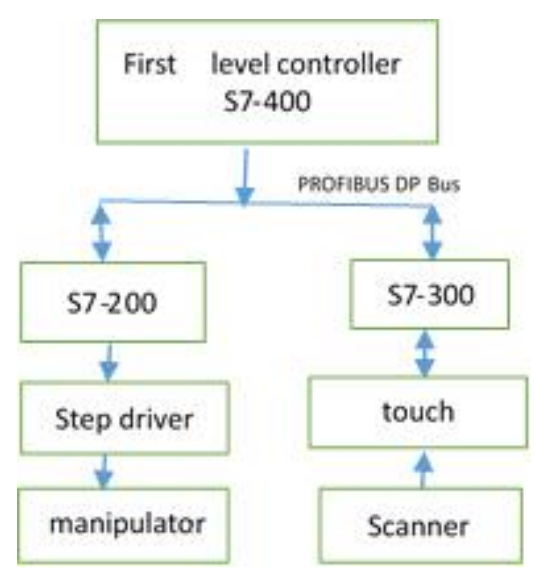

Fig. 1. System structure diagram

The mail sorting demonstration system mainly consists of three parts, the scan code detection, the controller and the actuator. Among them, the scan code detection part mainly uses the RFID radio frequency identification scan code to complete the inspection work, through the identification of the various places on the mail specific coding, distinguish different regions correspond to each sorting channel. Secondary sorting controller mainly by the Lord, from the station controller composition, Siemens S7-400 PLC as master controller[4]. It is responsible for collect and store all the information, by mail barcode to distinguish them, through the PROFIBUS-DP bus transfers to the secondary controller. Secondary control is made up of the SIEMENS S7-200/300PLC, which is mainly responsible for the distribution of the goods to be sorted two times. The goods are transferred to the manipulator part, the sorting part is the key part of the 4 degree of freedom manipulator, and it is mainly responsible for the different addresses of goods sorting to the corresponding storage location.

This design uses the Siemens S7 Series PLC. Among them, CPU- 412-2 DP PLC S7-400 as the main station It is a independent module, according to the needs of the design add input module for DI $32 \mathrm{DC} 24 \mathrm{~V}$, and output module for DO 32 DC24V/0.5A. SIEMENS S7-400 has a powerful control function and very fast computing power [6]. This can meet the requirements of the design of the master station. S7-200 224CN PLC as sub station 1, It has basic control function and operation ability. Sub station 2 select S7-300 CPU- 313C-2 DP PLC, which input 16 points, output 16 points, the model also has 1 MPI interface and 1 DP interface, it can complete the communication between the touch screen and SIEMENS S7300 through the MPI protocol. Usually pay attention to the PLC model and the order number is consistent; if not consistent will lead to hardware communication is not successful.

Touch screen is most commonly a human-computer interaction interface for monitoring industrial equipment, also known as human machine interface (HMI), It is the medium of transmitting and exchanging information between human and computer, and it is an important part of computer system. Among them, the touch screen as the front device of PLC set up a road between the user and machine; it uses simple and clear icons and symbols instead of traditional devices, such as an indicator, a selector switch, and so on. It saves cost and improves reliability.

The system uses the Taiwan Weinview Easy Builder8000 as the host computer monitoring software (hereinafter referred to as the EB8000), the monitoring system mainly completes sorting process of information acquisition and monitoring of the mail, so as to realize the sorting process control and reduce the workload

The execution part of the system is composed of stepping motor, stepping motor and mechanical hand. The driving machine adopts stepping motor to drive and control, so as to ensure the accuracy of the robot's movement. This design choose M415B type two phase hybrid stepping motor, it has high control precision, and when the power supply circuit is cut off, the stepper motor has a self positioning function, so it can keep the original position. Fig. 2 is a block diagram of the manipulator.

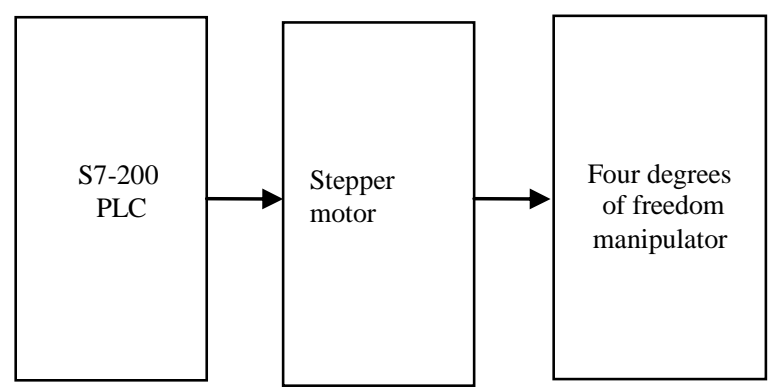

Fig. 2. Mechanical hand control block diagram

\section{B. Software design of simulation demonstration system}

According to the requirements of the design, simulation logistics sorting system in electricity, at all levels of the control center will return to set the initial position, then scan code guns for data acquisition, and the acquisition of encoded data entry into the database. After the data collection is completed, enter the main interface. The interface is processed at all levels of coded signal, and the signal at all levels is sent to all levels of the controller, the mail sorting to a specific location, complete the sorting of the logistics. It is shown in Fig.3. 


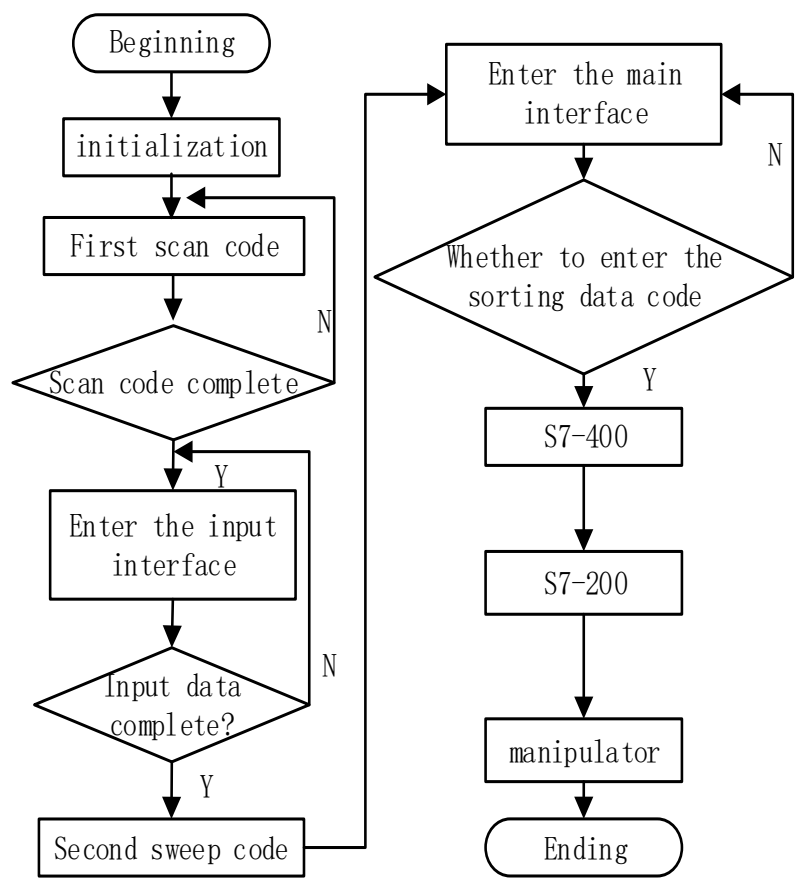

Fig. 3. PLC program flow chart

\section{COMMUNiCATION DESIGN OF SiMULATION DEMONSTRATION SYSTEM PREPARE YOUR PAPER BEFORE STYLING}

\section{A. Hardware Configuration of S7 Series PLC Based on Profibus}

Profibus belongs to the unit level and the field of SIMATIC network, it does not rely on the manufacturer's, open industrial field bus, can be used for distributed I/O device, transmission device and computer based automatic system[5]. The communication rate of PROFIBUS-DP is $19.2 \mathrm{kbit} / \mathrm{s} \sim 12 \mathrm{Mbit} / \mathrm{s}$, which is usually set to $1.5 \mathrm{Mbit} / \mathrm{s}$ by default, and the communication data packet is 244 bytes. Because of its fast data transmission, high transmission data density and good communication stability, it has become the mainstream of communication. This design uses the SIEMENS standard PROFIBUS-DP, its wiring method has many kinds, "A" terminal for the green line corresponds to the network interface "A", and also can "A" corresponding to the color of the red line "B". Network interface can be directly used to insert the PORT interface with the PLC, do not need to use intermediate equipment [6].

Through the SIEMENS programming software, the system establishes the communication between different PLC. Use the EM277 communication module to realize the communication between S7-200 and S7-400 by PROFIBUS-DP. It is shown as Fig.4. Based on the "online communication" function of the software, the communication between the PLC and the manmachine interface is completed. S7-300 uses the RS 485 communication line to complete the communication connection with the touch screen.

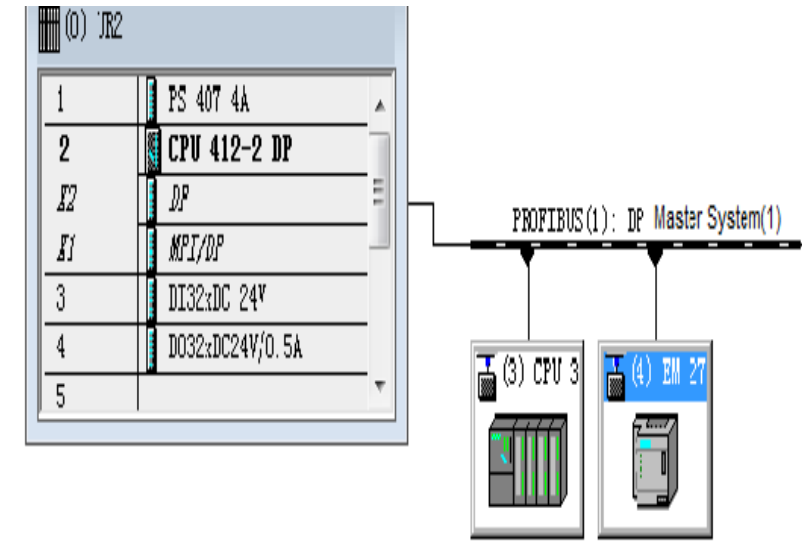

Fig. 4. System hardware configuration diagram

\section{B. Debugging and Running Result}

After the system completes the hardware design and installation, enter the system debugging. Mainly to detect the use of logistics sorting, through the man-machine interface to observe the actual process of sorting, and finally check the results of the design is in accordance with the requirements of logistics sorting process.

In the debugging of hardware system, first of all give power to the system, let master station PLC button from the "stop" dial to "run", secondary slave repeated master action, the corresponding indicator light, PLC operation, It is shown in Fig. 5 .

Siemens programming software with fault diagnosis function, when the program downloaded to the PLC and establish an online connection, open the module information can see the hardware fault information and programming error message, it will be easy to find the fault and solve.

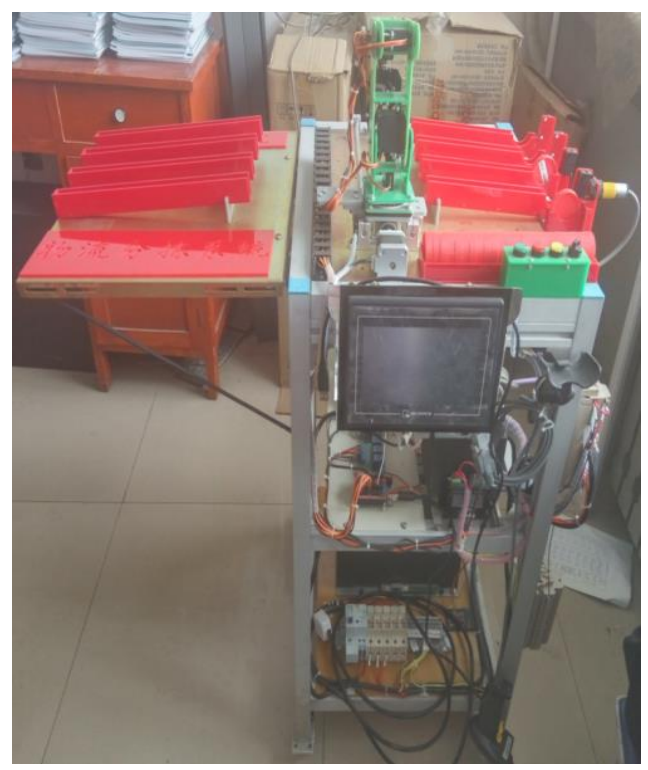

Fig. 5. Simulation system debugging 


\section{CONCLUSION}

This system uses the SIEMENS S7 series PLC and stepper motor, man-machine interface technology and RFID technology to combine each other, to achieve the simulation of the control and monitoring of logistics. The system solves the problem of the replacement of the controlled object, and the running process is vivid, and the reality is very strong. Therefore, students can solve the problem of learning PLC theory and the actual phase out of touch. And improve students' flexible use of PLC to solve problems, improve students' ability to design and create, give teachers and students intuitive understanding and help.

\section{ACKNOWLEDGMENT}

This research was performed with financial support from National college students' innovative training program under
Grant No.201510061067.and Teaching reform experimental teaching demonstration centre project of Tianjin Agricultural University under grant No.2015SY021.

\section{REFERENCES}

[1] Zhenxiang F., "The application of programming controller in mail sorting System," mechanical\&Electrical Engineering magazine.3,44-46(2000)

[2] DongSheng SH., Electrical control and programmable controller technology,Chemical Industry Press25-33,2003.

[3] Xia Ch., "Introduction and system design of programmable controller,"China Electric Power Press1, 42-50,2008.

[4] ZhaoJie Zh, TaiHe Fm, YuJu Ch. "SIEMENS S7-300 series PLC and application software Step7," South China University of Technology press, 38-49,2004.

[5] Ping Ch., ShuYue W., YanLun H.,"SIEMENS S7-300/400 rapid application," Posts \& Telecom Press, 8-10,2012.

[6] Gang Ch.and Ling L.,Huxiao dong."Automatic mail sorting system based on simens S7-200 series PLC,” Mechatronics3:87-89,2008. 